Article

\title{
Generation of an Adjustable Optical Cage through Focusing an Apertured Bessel-Gaussian Correlated Schell-Model Beam
}

\author{
Lina Guo ${ }^{1}$, Li Chen $^{1}$, Rong Lin ${ }^{2, *}$, Minghui Zhang ${ }^{3}$, Yaru Gao ${ }^{2}$ and Yangjian Cai ${ }^{2,4, *}$ \\ 1 School of Optoelectronic Engineering, Guangdong Polytechnic Normal University, Guangzhou 510665, \\ China; guoln523@126.com (L.G.); 13318903168@163.com (L.C.) \\ 2 Shandong Provincial Engineering and Technical Center of Light Manipulations \& Shandong Provincial Key \\ Laboratory of Optics and Photonic Device, School of Physics and Electronics, Shandong Normal University, \\ Jinan 250014, China; gaoyaru@siom.ac.cn \\ 3 School of Physics and Material Science, Anhui University, Hefei 230039, China; zmh@ahu.edu.cn \\ 4 School of Physical Science and Technology, Soochow University, Suzhou 215006, China \\ * Correspondence: hzxywlxlr@163.com (R.L.); yangjiancai@suda.edu.cn (Y.C.); Tel.: +86-531-89611187
}

Received: 11 January 2019; Accepted: 1 February 2019; Published: 7 February 2019

\begin{abstract}
An adjustable optical cage generated by focusing a partially coherent beam with nonconventional correlation function named the Bessel-Gaussian correlated Schell-model (BGCSM) beam is investigated in detail. With the help of the generalized Huygens-Fresnel integral and complex Gaussian function expansion, the analytical formula of the BGCSM beam passing through an apertured $A B C D$ optical system was derived. Our numerical results show that the generated optical cage can be moderately adjusted by the aperture radius, the spatial coherence width, and the parameter $\beta$ of the BGCSM beam. Furthermore, the effect of these parameters on the effective beam size and the spectral degree of coherence were also analyzed. The optical cage with adjustable size can be applied for particle trapping and material thermal processing.
\end{abstract}

Keywords: partially coherent beam; correlation function; focusing; optical cage

\section{Introduction}

The optical cage, also named the optical bottle beam (i.e., a three-dimensional dark spot surrounded by regions of higher intensity) has attracted growing attention. The optical cage has found wide applications in super-resolution fluorescence microscopy [1,2], dark optical traps for atoms [3,4] and microparticles [5], imaging very dim objects located reasonably close to bright objects [6]. Various techniques for generating the optical cage have been developed, such as two-beam interference [7-9], axicon [10], the spatial light modulator [11,12], and tight focusing of cylindrical vector beams [13,14]. Note that all the optical cages produced by the techniques mentioned above are completely coherent. A partially coherent optical cage may exhibit some advantages over those of a coherent optical cage, for example, a partially coherent optical cage is less sensitive to speckle [15-17]. Recently, there has been a growing interest in generating partially coherent optical cages. Several approaches have been developed to generate partially coherent 3D optical cages, such as the axicon-lens system [18] and the binary diffractive optical element [19]. Furthermore, controlling the spatial coherence of a focused partially coherent source has also shown the possibility of forming an optical cage in the focal region [20].

Recently, partially coherent beams with nonconventional correlation functions (NCFs) have been widely investigated [21,22]. Several types of partially coherent sources with NCFs have been introduced and generated through various coherence manipulation methods [23-40]. These novel sources exhibit many interesting propagation properties, and are expected to be useful in many 
applications, such as beam shaping, microscopy imaging, optical trapping, and free-space optical communications [35-38]. As two typical partially coherent beams with NCFs, the Laguerre-Gaussian correlated Schell-model (LGCSM) beam and the Bessel-Gaussian correlated Schell-model (BGCSM) beam were introduced in theory [27], and later generated in experiment with the aid of a spatial light modulator or with a hologram and a diffuser $[21,39,40]$. Their correlation functions are expressed in the form of Laguerre-Gaussian and Bessel-Gaussian functions, respectively. Both LGCSM and BGCSM beams display ring-shaped beam profiles in the far field in free space. It was also demonstrated that BGCSM and LGCSM beams have advantages over a partially coherent beam with a conventional correlation function (i.e., Gaussian Schell-model beam) to reduce turbulence-induced degeneration upon propagation in turbulent atmosphere [41,42], which will be beneficial in free-space optical communications. Furthermore, Chen et al. demonstrated both theoretically and experimentally that by focusing an LGCSM beam with a thin lens and tailoring the source correlation function, a controllable partially coherent optical cage can be generated near the focal area [43]. Thus, one may ask: can an adjustable optical cage be generated from focusing a BGCSM beam? In this paper, we demonstrate that a partially coherent optical cage can be generated by focusing an apertured BGCSM beam. We also show that the size and depth of the generated optical cage is adjustable by modulating the source spatial correlation function, the coherence width, and the aperture radius. The effective beam width and spectral degree of coherence (SDOC) of the focused BGCSM beam in the focal region are also discussed. Our results may find uses in optical trapping and material thermal processing.

\section{Propagation of an Apertural BGCSM Beam through a Paraxial ABCD Optical System}

The second-order correlation properties of a scalar partially coherent beam are generally characterized by the cross-spectral density (CSD) in the spatial-frequency domain [44]. The CSD of a BGCSM beam at two arbitrary points in the source plane is expressed as [27,40]:

$$
W\left(r_{1}, r_{2}\right)=\exp \left(-\frac{r_{1}^{2}+r_{2}^{2}}{4 \sigma_{0}^{2}}\right) \exp \left[-\frac{\left(r_{1}-r_{2}\right)^{2}}{2 \delta_{0}^{2}}\right] J_{0}\left(\beta \frac{\left|r_{1}-r_{2}\right|}{\delta_{0}}\right),
$$

where $\boldsymbol{r}_{j}=\left(\boldsymbol{r}_{j x}, \boldsymbol{r}_{j y}\right)(j=1,2)$ denotes an arbitrary transverse position vector, $\sigma_{0}$ is the transverse beam waist width of the corresponding Gaussian beam, $\delta_{0}$ is the transverse coherence width, $\beta$ is a real constant, and $J_{0}(x)$ is the zeroth-order Bessel function of the first kind. Equation (1) shows that the BGCSM beam reduces to a conventional Gaussian Schell-model beam when $\beta$ equals 0 , and to a $J_{0}$-correlated beam when $\beta$ trends towards infinity.

In a practical optical system, an aperture is usually used to modulate the size of the beam spot of a partially coherent beam. Therefore, it was necessary to investigate the effect of the aperture radius on the propagation properties of a partially coherent beam. We assumed that a circular aperture with radius $a$ is located in front of the BGCSM beam, so the CSD of the apertured BGCSM beam in the source plane is expressed as

$$
W\left(r_{1}, r_{2}\right)=\exp \left(-\frac{r_{1}^{2}+r_{2}^{2}}{4 \sigma_{0}^{2}}\right) \exp \left[-\frac{\left(r_{1}-r_{2}\right)^{2}}{2 \delta_{0}^{2}}\right] J_{0}\left(\beta \frac{\left|r_{1}-r_{2}\right|}{\delta_{0}}\right) H\left(r_{1}\right) H^{*}\left(r_{2}\right),
$$

where $H\left(\boldsymbol{r}_{i}\right)$ is the transmission function of the circular aperture. This can be expanded as the following finite sum of complex Gaussian functions [45,46]:

$$
H\left(r_{i}\right)=\sum_{m=1}^{M} A_{m} \exp \left(-\frac{B_{m} r_{i}^{2}}{a^{2}}\right) .
$$

Here, $A_{m}$ and $B_{m}$ are the expansion and Gaussian coefficients, respectively, which can be obtained through numerical optimization [45]. This expansion method has proved to be reliable and efficient, 
and the simulation accuracy improves as $M$ increases. For a circular hard aperture, $M=10$ already ensures a very good description of the propagation of the diffracted beam $[45,46]$.

The propagation of the CSD of an apertured BGCSM beam through a paraxial ABCD optical system can be studied by using the following extended Collins formula $[47,48]$ :

$$
\begin{aligned}
W\left(\boldsymbol{\rho}_{1}, \boldsymbol{\rho}_{2}\right)= & \left(\frac{k}{2 \pi B}\right)^{2} \exp \left[-\frac{i k D}{2 B}\left(\boldsymbol{\rho}_{1}^{2}-\boldsymbol{\rho}_{2}^{2}\right)\right] \int_{-\infty}^{\infty} \int_{-\infty}^{\infty} \int_{\infty}^{\infty} \int_{-\infty}^{\infty} W\left(\mathbf{r}_{1}, \mathbf{r}_{2}\right) \\
& \times \exp \left[-\frac{i k A}{2 B}\left(\mathbf{r}_{1}^{2}-\mathbf{r}_{2}^{2}\right)+\frac{i k}{B}\left(\mathbf{r}_{1} \cdot \boldsymbol{\rho}_{1}-\mathbf{r}_{2} \cdot \boldsymbol{\rho}_{2}\right)\right] d^{2} \mathbf{r}_{1} d^{2} \mathbf{r}_{2},
\end{aligned}
$$

where $\rho_{j}=\left(\rho_{j x}, \rho_{j y}\right)(j=1,2)$ denotes an arbitrary transverse position vector at the receiver plane and $k=2 \pi / \lambda$ is the wave number related to the wavelength $\lambda$.

For the convenience of integration, the following "sum" and "difference" coordinates were adopted:

$$
r_{s}=\frac{r_{1}+r_{2}}{2}, \Delta r=r_{1}-r_{2}, \rho_{s}=\frac{\rho_{1}+\rho_{2}}{2}, \Delta \rho=\rho_{1}-\rho_{2} .
$$

Substituting Equations (2) and (5) into Equation (4), we obtained the following alternative expression of Equation (4):

$$
\begin{aligned}
W\left(\boldsymbol{\rho}_{s}, \boldsymbol{\rho}_{d}\right)= & \left(\frac{k}{2 \pi B}\right)^{2} \exp \left[-\frac{i k D}{B} \boldsymbol{\rho}_{s} \cdot \Delta \boldsymbol{\rho}\right] \\
& \times \int_{-\infty}^{\infty} \int_{-\infty}^{\infty} \int_{\infty}^{\infty} \int_{-\infty}^{\infty} \sum_{m=1}^{N} \sum_{n=1}^{N} A_{m} A_{n}^{*} \exp \left[-\left(\frac{1}{2 \sigma_{0}^{2}}+\frac{B_{m}}{a^{2}}+\frac{B_{n}^{*}}{a^{2}}\right) \mathbf{r}_{s}^{2}\right] \\
& \times \exp \left[\left(\frac{i k}{B} \Delta \boldsymbol{\rho}-\frac{i k A}{B} \Delta \mathbf{r}-\frac{B_{m}}{a^{2}} \Delta \mathbf{r}+\frac{B_{n}^{*}}{a^{2}} \Delta \mathbf{r}\right) \cdot \mathbf{r}_{s}\right] d^{2} \mathbf{r}_{s} \\
& \times \exp \left[-\left(\frac{1}{8 \sigma_{0}^{2}}+\frac{1}{2 \delta_{0}^{2}}+\frac{B_{m}}{4 a^{2}}+\frac{B_{n}^{*}}{4 a^{2}}\right) \Delta \mathbf{r}^{2}\right] \\
& \times J_{0}\left(\beta \frac{|\Delta \mathbf{r}|}{\delta_{0}}\right) \exp \left[\frac{i k}{B} \boldsymbol{\rho}_{s} \cdot \Delta \mathbf{r}\right] d^{2} \Delta \mathbf{r}
\end{aligned}
$$

After integrating over $r_{\mathrm{s}}$ and $\Delta r$, we obtained the analytical cross-spectral density expression of an apertured BGCSM beam passing through a paraxial $A B C D$ optical system as follows:

$$
\begin{aligned}
W\left(\boldsymbol{\rho}_{s}, \boldsymbol{\rho}_{d}\right)= & \left(\frac{k}{2 B}\right)^{2} \exp \left[-\frac{i k D}{B} \boldsymbol{\rho}_{s} \cdot \Delta \boldsymbol{\rho}\right] \sum_{m=1}^{N} \sum_{n=1}^{N} \frac{A_{m} A_{n}^{*}}{\zeta \gamma} \exp \left(-\frac{\beta^{2}}{4 \delta_{0}{ }^{2} \gamma}-\frac{k^{2}}{4 \varsigma B^{2}} \Delta \boldsymbol{\rho}^{2}\right) \\
& \times \exp \left(-\frac{1}{4 \gamma}\left|\frac{k}{B} \boldsymbol{\rho}_{s}-\frac{k}{2 B \zeta}\left(\frac{i k A}{B}+\frac{B_{m}}{a^{2}}-\frac{B_{n}^{*}}{a^{2}}\right) \Delta \boldsymbol{\rho}\right|^{2}\right) \\
& \times I_{0}\left(\frac{\beta}{2 \delta_{0} \gamma}\left|\frac{k}{B} \boldsymbol{\rho}_{s}-\frac{k}{2 B \zeta}\left(\frac{i k A}{B}+\frac{B_{m}}{a^{2}}-\frac{B_{n}^{*}}{a^{2}}\right) \Delta \boldsymbol{\rho}\right|\right),
\end{aligned}
$$

with

$$
\varsigma=\frac{1}{2 \sigma_{0}^{2}}+\frac{B_{m}}{a^{2}}+\frac{B_{n}^{*}}{a^{2}}, \quad \gamma=\frac{\varsigma}{4}+\frac{1}{2 \delta_{0}^{2}}-\frac{1}{4 \zeta}\left(\frac{i k A}{B}+\frac{B_{m}}{a^{2}}-\frac{B_{n}^{*}}{a^{2}}\right)^{2} .
$$

In the above derivations, we applied the following integral formulae [49]:

$$
\begin{gathered}
\int_{-\infty}^{\infty} \exp \left(-p^{2} x^{2} \pm q x\right) d x=\frac{\sqrt{\pi}}{p} \exp \left(\frac{q^{2}}{4 p^{2}}\right), \quad(p>0), \\
J_{v}(\chi)=\frac{(-i)^{v}}{2 \pi} \int_{0}^{2 \pi} \exp [i v \theta+i \chi \cos (\theta)] d \theta, \\
\int_{0}^{\infty} x \exp \left(-\gamma x^{2}\right) J_{v}(p x) J_{v}(q x) d x=\frac{1}{2 \gamma} \exp \left(\frac{p^{2}+q^{2}}{4 \gamma}\right) I_{v}\left(\frac{p q}{2 \gamma}\right),[\operatorname{Re} \gamma>0, \operatorname{Re} v>-1],
\end{gathered}
$$

where $J_{v}(x)$ and $I_{v}(x)$ are the $v$-order Bessel and modified Bessel functions of the first kind, respectively. 
Accordingly, the spectral density of the BGCSM beam in the receiver plane was obtained as:

$$
I(\boldsymbol{\rho})=W(\boldsymbol{\rho}, 0)=\left(\frac{k}{2 B}\right)^{2} \sum_{m=1}^{N} \sum_{n=1}^{N} \frac{A_{m} A_{n}^{*}}{\gamma \zeta} \exp \left(-\frac{k^{2}}{\gamma B^{2}}|\boldsymbol{\rho}|^{2}-\frac{\beta^{2}}{4 \delta_{0}^{2} \gamma}\right) I_{0}\left(\frac{k \beta|\boldsymbol{\rho}|}{\delta_{0} B \gamma}\right)
$$

In the cylindrical coordinates, the effective beam width of a circular symmetric beam is defined as [50-52]:

$$
w=\sqrt{\frac{2 \int_{-\infty}^{\infty} \rho^{2} I(\rho) d^{2} \rho}{\int_{-\infty}^{\infty} I(\rho) d^{2} \rho}} .
$$

Applying the following expansion formula:

$$
I_{v}[x]=\sum_{s=0}^{\infty} \frac{x^{v+2 s}}{s ! \Gamma(v+s+1) 2^{v+2 s}}
$$

then, substituting Equations (12) and (14) into Equation (13), we obtained the effective beam width for an aperture BGCSM beam through a paraxial ABCD optical system as follows:

$$
w=\sqrt{\frac{2 F_{1}}{F_{2}}}
$$

with

$$
\begin{gathered}
F_{1}=\sum_{m=1}^{N} \sum_{n=1}^{N} \sum_{s=0}^{\infty} \frac{A_{m} A_{n}^{*} b^{2 s}(s+1)}{\gamma \varsigma s ! 2^{2 s} \alpha^{2+s}} \exp \left(\frac{\beta^{2}}{4 \delta_{0}^{2} \gamma}\right), \\
F_{2}=\sum_{m=1}^{N} \sum_{n=1}^{N} \sum_{s=0}^{\infty} \frac{A_{m} A_{n}^{*} b^{2 s}}{\gamma \varsigma s ! 2^{2 s} \alpha^{s+1}} \exp \left(\frac{\beta^{2}}{4 \delta_{0}^{2} \gamma}\right),
\end{gathered}
$$

where

$$
\alpha=\frac{k^{2}}{4 \gamma B^{2}}, b=\frac{k \beta}{2 \delta_{0} B \gamma} .
$$

The SDOC of an apertured BGCSM beam between two arbitrary points $\rho_{1}$ and $\rho_{2}$ in the receiver plane was obtained as:

$$
\mu\left(\rho_{1}, \rho_{2}\right)=\frac{W\left(\frac{\rho_{1}+\rho_{2}}{2}, \rho_{1}-\rho_{2}\right)}{\sqrt{W\left(\rho_{1}, 0\right) W\left(\rho_{2}, 0\right)}} .
$$

\section{Numerical Calculation Results}

Considering an apertured BGCSM beam generating from the source plane $(z=0)$ focused by a thin lens optical system with a focal length $f$, the distance from the source plane to the thin lens is $f$ and the distance from the thin lens to the receiver plane is $z$. Thus, the transfer matrix for the optical system can be expressed as:

$$
\left(\begin{array}{ll}
A & B \\
C & D
\end{array}\right)=\left(\begin{array}{ll}
1 & z \\
0 & 1
\end{array}\right)\left(\begin{array}{cc}
1 & 0 \\
-1 / f & 1
\end{array}\right)\left(\begin{array}{ll}
1 & f \\
0 & 1
\end{array}\right)=\left(\begin{array}{cc}
1-\frac{z}{f} & f \\
-1 / f & 1
\end{array}\right) .
$$

We studied numerically focusing characteristic of the intensity, the effective beam width, and the SDOC of an apertured BGCSM beam focused by a thin lens focusing system by applying the formulae derived in Section 2. For the following numerical examples, the parameters were chosen as $f=150 \mathrm{~mm}, \lambda=632.8 \mathrm{~mm}, \sigma_{0}=1 \mathrm{~mm}, a=1.5 \mathrm{~mm}, \delta_{0}=0.2 \mathrm{~mm}$, and $\beta=2.5$, unless other values are indicated in the figures.

To study the intensity properties of an apertured BGCSM beam focused by a thin lens focusing system, in Figures 1-3 we calculated the normalized intensity distribution of an apertured BGCSM 
beam in the $\rho-z$ plane for different values of the aperture radius $a$, the transverse coherence width $\delta_{0}$, and the parameter $\beta$, respectively. One can see from Figure 1 that a partially coherent optical cage was formed with or without an aperture separately. The shape of the optical cage became much more uniform and the size of the cage became larger with the decrease of the aperture radius. The generated optical cage is useful for trapping low-refractive index particles. As shown in Figure 2, with the small value of $\delta_{0}$, a more uniform optical cage was formed. With the increase of the coherence width $\delta_{0}$, both transverse and longitudinal sizes, as well as the depth of the optical cage, decreased. As shown in Figure 3, with the increase of the parameter $\beta$, the focal intensity profile gradually evolved from a peak-centered shape into an optical cage, and both transverse and longitudinal sizes, as well as the depth of the optical cage increased. However, it was also found that, with the increase of the parameter $\beta$, the transverse intensity surrounding the optical cage increased while the longitudinal intensity surrounding the optical cage decreased, so the shape of the optical cage became less uniform. Hence, a $J_{0}$-correlated beam (i.e., a BGCSM beam with $\beta \rightarrow \infty$ ) is not appropriate for producing an optical cage. From Figures 2 and 3, one can explain that the effect of the special correlation function can modulate the optical cage and will be enhanced with a small $\delta_{0}$ or a moderate $\beta$. Furthermore, we conclude that one can conveniently obtain an adjustable optical cage by focusing an aperture BGCSM beam through choosing appropriate aperture radius, transverse coherence width, and correlation function (i.e., the Bessel-Gaussian correlation function). However, the conventional Gaussian Shell-model beams do not have those characteristics.
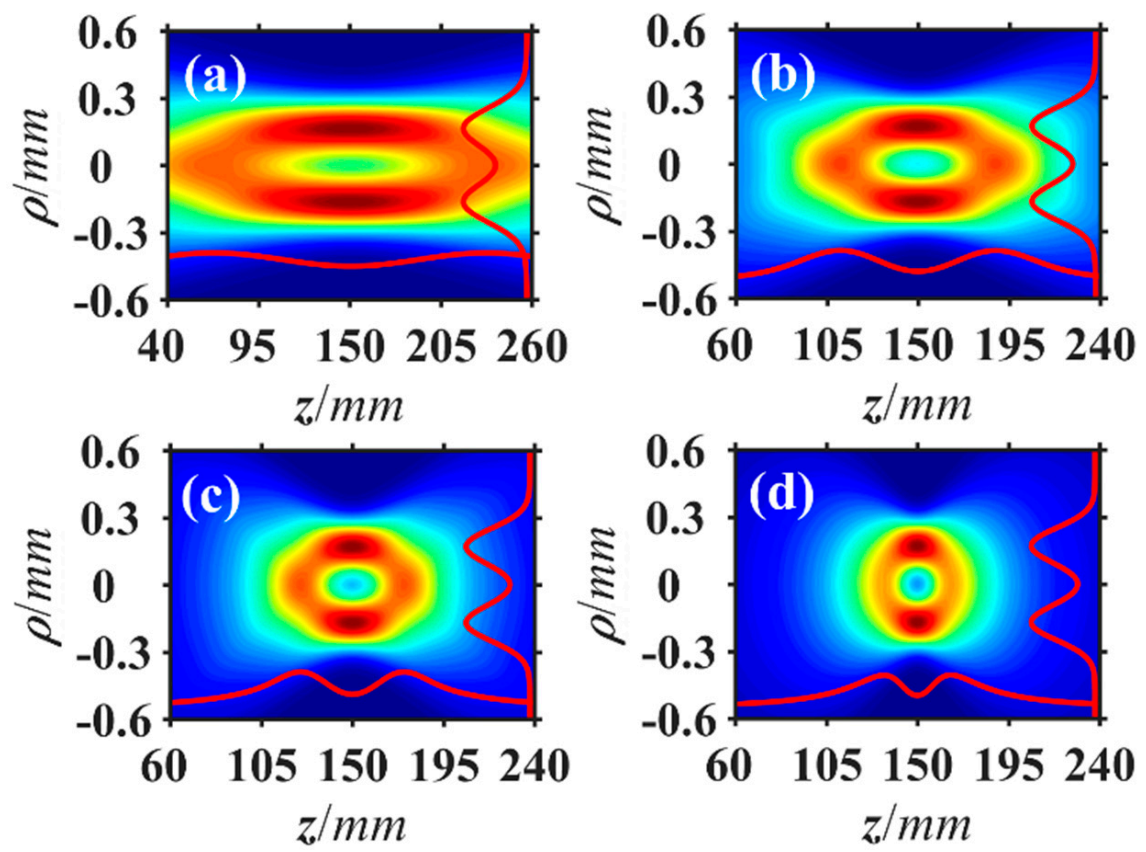

Figure 1. Normalized intensity distribution of an apertured Bessel-Gaussian correlated Schell-model (BGCSM) beam focused by a thin lens in the $\rho-z$ plane for different values of aperture radius $a$, with $\delta_{0}$ $=0.2 \mathrm{~mm}$ and $\beta=2.5$. (a) $a=0.5 \mathrm{~mm}$; (b) $a=1 \mathrm{~mm}$; (c) $a=1.5 \mathrm{~mm}$; and (d) without aperture. 

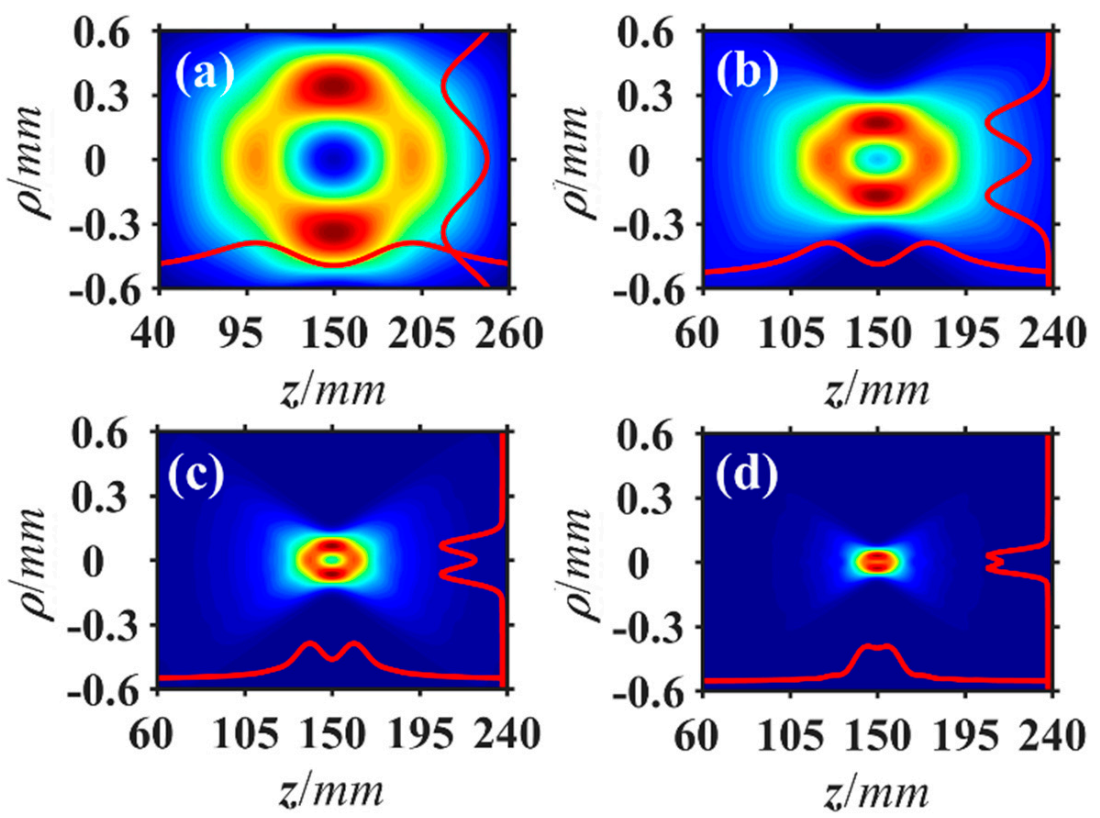

Figure 2. Normalized intensity distribution of an apertured BGCSM beam focused by a thin lens in the $\rho-z$ plane for different values of transverse coherence width $\delta_{0}$ with $a=1.5 \mathrm{~mm}$ and $\beta=2.5$. (a) $\delta_{0}=0.1$ $\mathrm{mm}$; (b) $\delta_{0}=0.2 \mathrm{~mm}$; (c) $\delta_{0}=0.5 \mathrm{~mm}$; and (d) $\delta_{0}=1 \mathrm{~mm}$.
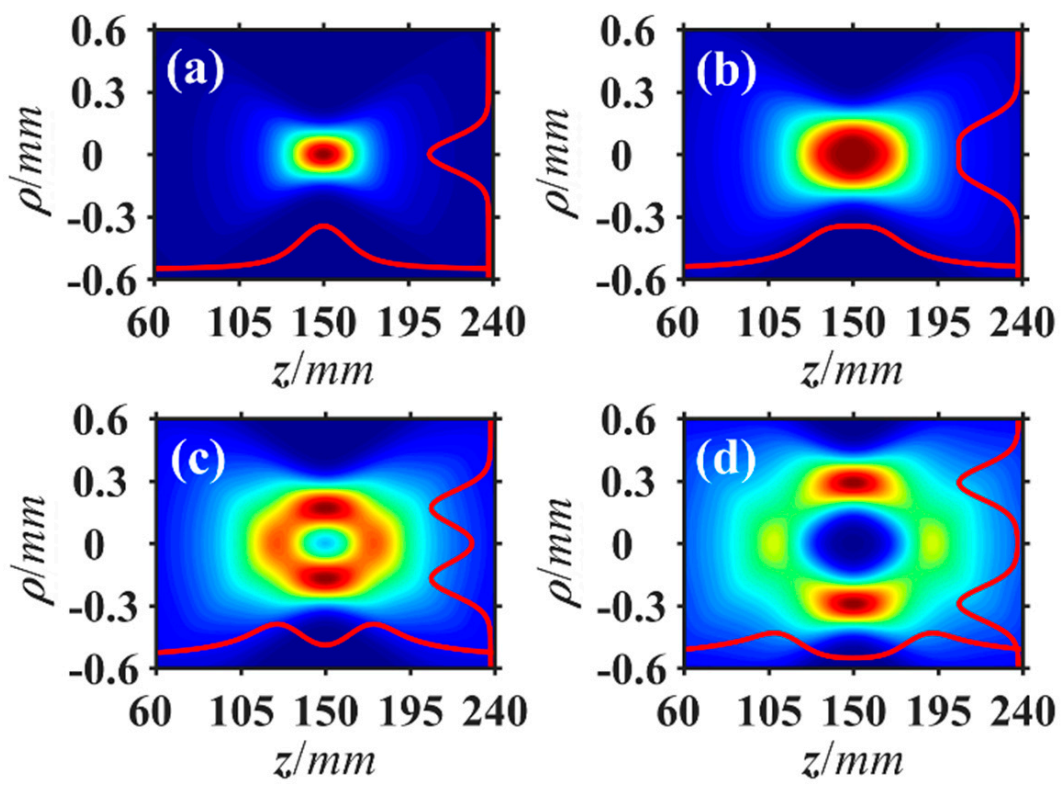

Figure 3. Normalized intensity distribution of an apertured BGCSM beam focused by a thin lens in the $\rho-z$ plane for different values of $\beta$ with $a=1.5 \mathrm{~mm}$ and $\delta_{0}=0.2 \mathrm{~mm}$. (a) $\beta=0$; (b) $\beta=1.5$; (c) $\beta=2.5$; and (d) $\beta=4$.

Next, let us study the effective beam width of an apertured BGCSM beam. The effective beam width of an apertured BGCSM beam in the focal plane for different values of the aperture radius $a$, the transverse coherence width $\delta_{0}$, and the parameter $\beta$ are depicted in Figure 4 . From Figure $4 \mathrm{a}$, we can see that with the increase of the aperture radius $a$, the effective beam width first quickly decreased and then tended toward a minimum value, which was the effective beam width of the unapertured BGCSM beam in the focal plane. Additionally, the effective beam width increased with the increase of $\beta$. From Figure $4 b$, we can see that if the aperture radius $a$ kept invariant, with the increase of the 
coherence width $\delta_{0}$, the effective beam width first quickly decreased and then approached a common value, which was independent of the coherence width. This common value can be considered as the effective beam width of a coherent Gaussian beam. As seen from Figure $4 c$, the effective beam width increased as the parameter $\beta$ increased, which is consistent with Figure 4a. For a large value of $a$ (i.e., $a>1.5 \mathrm{~mm}$ ), the effective beam width was nearly unchanged, so the effect of an aperture can be neglected.

(a)

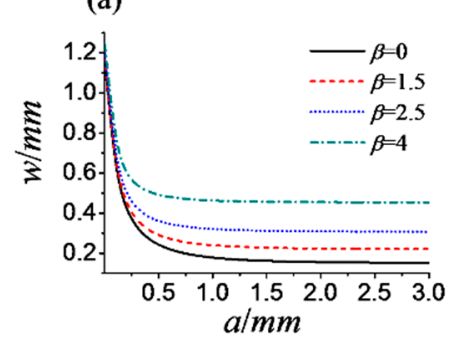

(b)

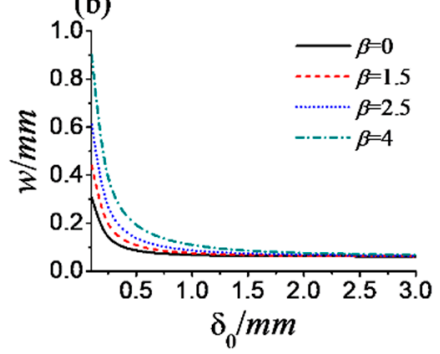

(c)

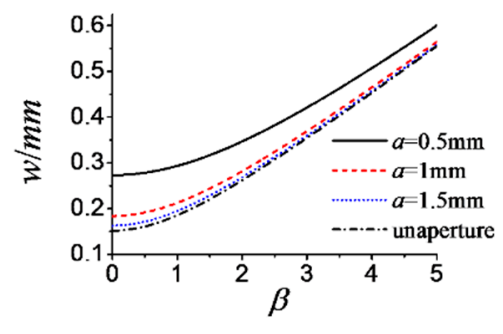

Figure 4. Effective beam waist width of an apertured BGCSM beams in the focal plane versus (a) aperture radius $a,(\mathbf{b})$ transverse coherence width $\delta_{0}$, and (c) $\beta$. The other parameters were set as $\delta_{0}=$ $0.2 \mathrm{~mm}$ in (a), $a=1.5 \mathrm{~mm}$ in (b), and $\delta_{0}=0.2 \mathrm{~mm}$ in (c) .

Finally, we discuss the SDOC of an apertured BGCSM beam focused by a thin lens. Figure 5 shows the modulus of the SDOC $|\mu(\rho, 0)|$ of an apertured BGCSM beam at two transverse spatial positions, $\rho_{1}=\rho$ and $\rho_{2}=\mathbf{0}$, propagating in the $\rho-z$ plane for different values of the aperture radius $a$. One sees clearly that the $|\mu(\rho, 0)|$ displayed a non-Gaussian distribution with two side robes around the central bright spot on propagation, and finally degenerated into a Gaussian distribution in the focal plane when the aperture disappeared. While there was an existing aperture, with the decrease of $a,|\mu(\rho, 0)|$ diverged more quickly on propagation, and displayed non-Gaussian distribution with more side robes in the focal region.
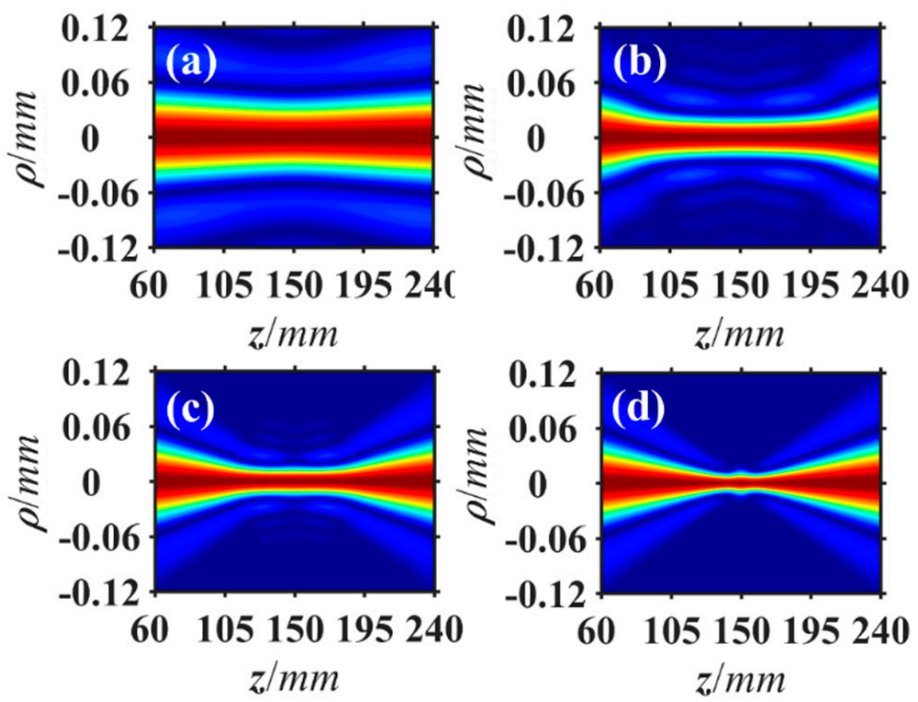

Figure 5. Modulus of the spectral degree of coherence (SDOC) $|\mu(\rho, 0)|$ of an apertured BGCSM beam focused by a thin lens in the $\rho-z$ plane for different $a$, with $\beta=2.5$ and $\delta_{0}=0.2 \mathrm{~mm}$. (a) $a=0.5$; (b) $a=1$; (c) $a=1.5 ;$ and (d) without aperture.

To further study the SDOC in the focal plane, in Figure 6 we calculated the modulus of the SDOC $|\mu(\rho, 0)|$ in the focal plane between $\rho_{1}=\boldsymbol{\rho}$ and $\rho_{2}=\mathbf{0}$ for different values of the aperture radius $a$, 
the transverse coherence width $\delta_{0}$, and the parameter $\beta$, respectively. One finds from Figure $6 \mathrm{a}$ that without aperture, $|\mu(\rho, 0)|$ exhibited a Gaussian distribution in the focal plane. However, with the decrease of $a,|\mu(\rho, 0)|$ exhibited several zero points. These zero points indicate that both real and imaginary parts of $|\mu(\rho, 0)|$ equal 0 , and thus the correlation singularities were formed [53,54]. These correlation singularities were caused by the existence of the aperture, since no correlation singularities existed in the case without aperture. In addition, with the decrease of $a$, the value of $|\mu(\rho, 0)|$ decreased and more correlation singularities were formed. From Figure $6 \mathrm{~b}$,c, we can see that the value of $|\mu(\rho, 0)|$ increased with the increase of $\delta_{0}$ or decrease of $\beta$. The numbers of correlation singularities were not affected by $\delta_{0}$ and $\beta$, while their locations were affected by $\delta_{0}$ and $\beta$.
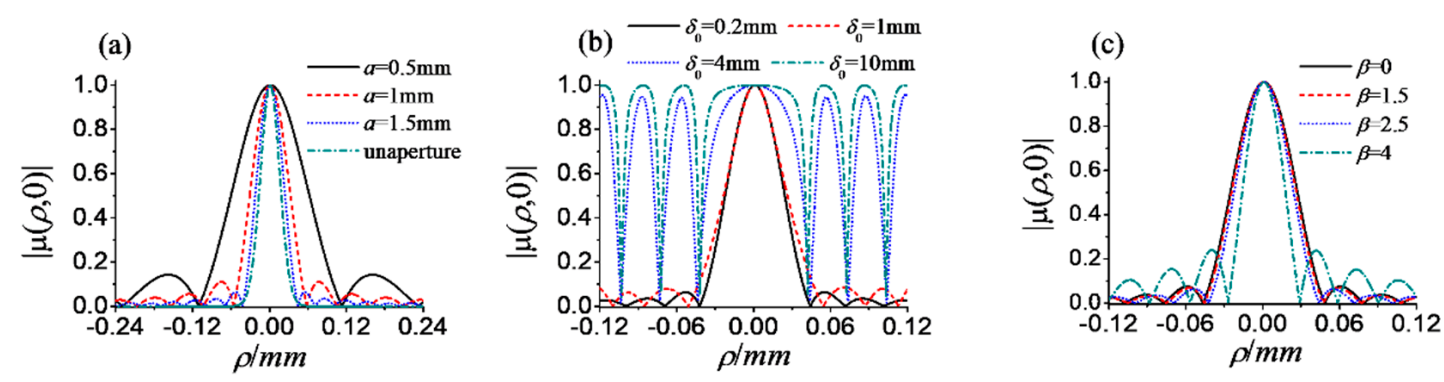

Figure 6. Dependence of modulus of the SDOC $|\mu(\rho, 0)|$ of apertured BGCSM beams in the focal plane on (a) the normalized truncation parameter $a,(\mathbf{b})$ the transverse coherence width $\delta_{0}$, and (c) the parameter $\beta$. The other parameters were set as $\delta_{0}=0.2 \mathrm{~mm}$ and $\beta=2.5 \mathrm{in}(\mathbf{a}), a=1.5 \mathrm{~mm}$ and $\beta=2.5 \mathrm{in}$ (b), and $a=1.5 \mathrm{~mm}$ and $\delta_{0}=0.2 \mathrm{~mm}$ in (c).

\section{Conclusions}

The statistical properties of an apertured BGCSM beam focused by a thin lens have been explored. The adjustable size of a formed optical cage is the most attractive property. We found that the optical cage could be controlled by manipulating the initial spatial coherence width $\delta_{0}$, the parameter $\beta$, and the aperture radius $a$. The size and depth of the optical cage increased with the decrease of $\delta_{0}$ or increase of $\beta$. The shape of the optical cage became much more uniform with small values of $a$ and $\delta_{0}$, or a moderate value of $\beta$. In addition, the effective beam width and the SDOC were also affected by these parameters. Our results will be rewarding in various applications, such as in particle trapping, dark field microscopy, and super-resolution fluorescence microscopy.

Author Contributions: L.G. (Data curation, Writing—original draft, Methodology); L.C. (Formal analysis, Writingreview and editing); R.L. (Methodology, Writing-review and editing); M.Z. (Formal analysis, Writing-review and editing), Y.G. (Formal analysis, Writing-review and editing), Y.C. (Supervision, Project administration, Writing-review and editing).

Funding: This research was funded by the National Natural Science Fund for Distinguished Young Scholar [11525418], the National Natural Science Foundation of China [91750201], Characteristic Innovation Project (Natural Science) of the Education Department of Guangdong Province [2017KTSCX114].

Conflicts of Interest: The authors declare no conflict of interest.

\section{References}

1. Harke, B.; Ullal, C.K.; Keller, J.; Hell, S.W. Three-dimensional nanoscopy of colloidal crystals. Nano Lett. 2008, 8, 1309-1313. [CrossRef] [PubMed]

2. Watanabe, T.; Iketaki, Y.; Omatsu, T.; Yamamoto, K.; Sakai, M.; Fujii, M. Two-point-separation in super-resolution fluorescence microscope based on up-conversion fluorescence depletion technique. Opt. Express 2003, 11, 3271-3276. [CrossRef] [PubMed]

3. Ozeri, R.; Khaykovich, L.; Davidson, N. Long spin relaxation times in a single-beam blue-detuned optical trap. Phys. Rev. A 1999, 59, R1750-R1753. [CrossRef] 
4. Wang, J.; Zhan, M.; Xu, P.; He, X. Trapping a single atom in a blue detuned optical bottle beam trap. Opt. Lett. 2010, 35, 2164-2166. [CrossRef]

5. Gahagan, K.T.; Swartzlander, G.A. Optical vortex trapping of particles. Opt. Lett. 1996, 21, 827-829. [CrossRef] [PubMed]

6. Foo, G.; Palacios, D.M.; Swartzlander, G.A. Optical vortex coronagraph. Opt. Lett. 2005, 30, 3308-3310. [CrossRef] [PubMed]

7. Arlt, J.; Padgett, M.J. Generation of a beam with a dark focus surrounded by regions of higher intensity: The optical bottle beam. Opt. Lett. 2000, 25, 191-193. [CrossRef]

8. Chen, Z.; Zhao, D. 4Pi focusing of spatially modulated radially polarized vortex beams. Opt. Lett. 2012, 37, 1286-1288. [CrossRef]

9. Bouma, B.E.; Yelin, D.; Tearney, G.J. Generating an adjustable three-dimensional dark focus. Opt. Lett. 2004, 29, 661-663. [CrossRef]

10. Du, T.; Wang, T.; Wu, F. Generation of three-dimensional optical bottle beams via focused non-diffracting Bessel beam using an axicon. Opt. Commun. 2014, 317, 24-28. [CrossRef]

11. Nándor, B.; Davidson, N. Tight parabolic dark spot with high numerical aperture focusing with a circular $\pi$ phase plate. Opt. Commun. 2007, 270, 145-150. [CrossRef]

12. Mcgloin, D.; Spalding, G.C.; Melville, H.; Sibbett, W.; Dholakia, K. Three-dimensional arrays of optical bottle beams. Opt. Commun. 2003, 225, 215-222. [CrossRef]

13. Wang, X.L.; Ding, J.; Qin, J.Q.; Chen, J.; Fan, Y.X.; Wang, H.T. Configurable three-dimensional optical cage generated from cylindrical vector beams. Opt. Commun. 2009, 282, 3421-3425. [CrossRef]

14. Zhang, Y.; Ding, B.; Suyama, T. Trapping two types of particles using a double-ring-shaped radially polarized beam. Phys. Rev. A 2010, 81, 023831. [CrossRef]

15. Gbur, G.; Visser, T.D. Can spatial coherence effects produce a local minimum of intensity at focus? Opt. Lett. 2003, 28, 1627-1629. [CrossRef] [PubMed]

16. Gaarde, M.B.; Schafer, K.J. Generating single attosecond pulses via spatial filtering. Opt. Lett. 2006, 31, 3188-3190. [CrossRef] [PubMed]

17. Zhao, C.; Cai, Y.; Wang, F.; Lu, X.; Wang, Y. Generation of a high-quality partially coherent dark hollow beam with a multimode fiber. Opt. Lett. 2008, 33, 1389-1391. [CrossRef]

18. Pu, J.; Dong, M.; Wang, T. Generation of adjustable partially coherent bottle beams by use of an axicon-lens system. Appl. Opt. 2006, 45, 7553-7556. [CrossRef]

19. Shu, J.; Chen, Z.; Pu, J.; Zhu, J.; Liu, D. Tight focusing of partially coherent and radiallly polarized vortex beams. Opt. Commun. 2013, 295, 5-10. [CrossRef]

20. Pu, J.; Liu, X.; Nemoto, S. Partially coherent bottle beams. Opt. Commun. 2005, 252, 7-11. [CrossRef]

21. Cai, Y.; Chen, Y.; Wang, F. Generation and propagation of partially coherent beams with nonconventional correlation functions: A review[invited]. J. Opt. Soc. Am. A 2014, 31, 2083-2096. [CrossRef] [PubMed]

22. Cai, Y.; Chen, Y.; Yu, J.; Liu, X.; Liu, L. Generation of Partially Coherent Beams. Prog. Opt. 2017, 62, $157-223$. [CrossRef]

23. Chen, Y.; Ponomarenko, S.A.; Cai, Y. Experimental generation of optical coherence lattices. Appl. Phys. Lett. 2016, 109, 7085. [CrossRef]

24. Chen, Y.; Ponomarenko, S.A.; Cai, Y. Self-steering partially coherent beams. Sci. Rep. 2017, 7, 39957. [CrossRef]

25. Liu, X.; Liu, L.; Peng, X.; Liu, L.; Wang, F.; Gao, Y.; Cai, Y. Partially coherent vortex beam with periodical coherence properties. J. Quant. Spect. Rad. Trans. 2019, 222, 138-144. [CrossRef]

26. Liang, C.; Wang, F.; Liu, X.; Cai, Y.; Korotkova, O. Experimental generation of cosine-Gaussian-correlated Schell-model beams with rectangular symmetry. Opt. Lett. 2014, 39, 769-772. [CrossRef]

27. Mei, Z.; Korotkova, O. Random sources generating ring-shaped beams. Opt. Lett. 2013, 38, 91-93. [CrossRef] [PubMed]

28. Liang, C.; Zhu, X.; Mi, C.; Peng, X.; Wang, F.; Cai, Y.; Ponomarenko, S.A. High-quality partially coherent Bessel beam array generation. Opt. Lett. 2018, 43, 3188-3191. [CrossRef]

29. Zhang, Y.; Ding, C.; Pan, L.; Cai, Y. Laser arrays of partially coherent beams with multi-Gaussian correlation function. J. Quant. Spect. Rad. Trans. 2018, 218, 1-11. [CrossRef]

30. Peng, X.; Liu, L.; Wang, F.; Popov, S.; Cai, Y. Twisted Laguerre-Gaussian Schell-model beam and its orbital angular moment. Opt. Express 2018, 26, 33956-33969. [CrossRef] 
31. Piquero, G.; Santarsiero, M.; Martínez-Herrero, R.; de Sande, J.C.G.; Alonzo, M.; Gori, F. Partially coherent sources with radial coherence. Opt. Lett. 2018, 43, 2376-2379. [CrossRef]

32. Santarsiero, M.; Martínez-Herrero, R.; Maluenda, D.; de Sande, J.C.G.; Piquero, G.; Gori, F. Synthesis of circularly coherent sources. Opt. Lett. 2017, 42, 4115-4118. [CrossRef] [PubMed]

33. Yu, J.; Cai, Y.; Gbur, G. Rectangular Hermite non-uniformly correlated beams and its propagation properties. Opt. Express 2018, 26, 27894-27906. [CrossRef] [PubMed]

34. Liu, X.; Liu, L.; Wang, F.; Cai, Y. Generation of a flexible far-field anomalous hollow beam spot through superposition of two partially coherent sources with different degrees of coherence. Opt. Commun. 2018, 428, 69-76. [CrossRef]

35. Yu, J.; Wang, F.; Liu, L.; Cai, Y.; Gbur, G. Propagation properties of Hermite non-uniformly correlated beams in turbulence. Opt. Express 2018, 26, 16333-16343. [CrossRef] [PubMed]

36. Liang, C.; Wu, G.; Wang, F.; Li, W.; Cai, Y.; Ponomarenko, S.A. Overcoming the classical rayleigh diffraction limit by controlling two-point correlations of partially coherent light sources. Opt. Express 2017, 25, 28352-28362. [CrossRef]

37. Yuan, Y.; Liu, X.; Wang, F.; Chen, Y.; Cai, Y.; Qu, J.; Eyyuboğlu, H.T. Scintillation index of a multi-Gaussian Schell-model beam in turbulent atmosphere. Opt. Commun. 2013, 305, 57-65. [CrossRef]

38. Dao, W.; Liang, C.; Wang, F.; Cai, Y.; Hoenders, B.J. Effects of anisotropic turbulence on propagation characteristics of partially coherent beams with spatially varying coherence. Appl. Sci. 2018, 8, 2025. [CrossRef]

39. Reddy, S.G.; Kumar, A.; Prabhakar, S.; Singh, R.P. Experimental generation of ring-shaped beams with random sources. Opt. Lett. 2013, 38, 4441-4444. [CrossRef]

40. Wang, F.; Liu, X.; Yuan, Y.; Cai, Y. Experimental generation of partially coherent beams with different complex degrees of coherence. Opt. Lett. 2013, 38, 1814-1816. [CrossRef]

41. Chen, R.; Liu, L.; Zhu, S.; Wu, G.; Wang, F.; Cai, Y. Statistical properties of a Laguerre-Gaussian Schell-model beam in turbulent atmosphere. Opt. Express 2014, 22, 1871-1883. [CrossRef] [PubMed]

42. Wang, X.; Yao, M.; Qiu, Z.; Yi, X.; Liu, Z. Evolution properties of Bessel-Gaussian Schell-model beams in non-Kolmogorov turbulence. Opt. Express 2015, 23, 12508-12523. [CrossRef]

43. Chen, Y.; Cai, Y. Generation of a controllable optical cage by focusing a Laguerre-Gaussian correlated Schell-model beam. Opt. Lett. 2014, 39, 2549-2552. [CrossRef] [PubMed]

44. Munro, P.R.T. Introduction to the Theory of Coherence and Polarization of Light; Wolf, E., Ed.; Cambridge University Press: Cambridge, UK, 2007; ISBN 9780521822114.

45. Wen, J.J.; Breazeale, M.A. A diffraction beam field expressed as the superposition of Gaussian beams. J. Acoust. Soc. Am. 1988, 83, 1752-1756. [CrossRef]

46. Cai, Y.; Hu, L. Propagation of partially coherent twisted anisotropic Gaussian Schell-model beams through an apertured astigmatic optical system. Opt. Lett. 2006, 31, 685-687. [CrossRef] [PubMed]

47. Collins, S.A. Lens-system diffraction integral written in terms of matrix optics. J. Opt. Soc. Am. A 1970, 60, 1168-1177. [CrossRef]

48. Lin, Q.; Cai, Y. Tensor ABCD law for partially coherent twisted anisotropic Gaussian-Schell model beams. Opt. Lett. 2002, 27, 216-218. [CrossRef]

49. Gradshteyn, I.S.; Ryzhik, I.M. Table of Integrals, Series, and Products, 7th ed.; Jeffrey, A., Zwillinger, D., Eds.; Academic Press: London, UK, 2007; ISBN 9780123736376.

50. Carter, W.H. Spot size and divergence for Hermite Gaussian beams of any order. Appl. Opt. 1980, 19, 1027-1029. [CrossRef]

51. Siegman, A.E. New developments in laser resonators. Opt. Resonators 1990, 1224, 2-14. [CrossRef]

52. Yuan, Y.; Cai, Y.; Qu, J.; Eyyuboğlu, H.T.; Baykal, Y. Average intensity and spreading of an elegant Hermite-Gaussian beam in turbulent atmosphere. Opt. Express 2009, 17, 11130-11139. [CrossRef]

53. Gbur, G.; Visser, T.D. Coherence vortices in partially coherent beams. Opt. Commun. 2003, 222, 117-125. [CrossRef]

54. Schouten, H.F.; Gbur, G.; Visser, T.D.; Wolf, E. Phase singularities of the coherence functions in Young's interference pattern. Opt. Lett. 2003, 28, 968-970. [CrossRef] [PubMed]

(C) 2019 by the authors. Licensee MDPI, Basel, Switzerland. This article is an open access article distributed under the terms and conditions of the Creative Commons Attribution (CC BY) license (http:/ / creativecommons.org/licenses/by/4.0/). 